\title{
CONSTRUCTING PAIRS OF PANTS
}

\author{
Ara Basmajian
}

In this paper we supply necessary and sufficient conditions for two hyperbolic elements $(\gamma, \beta)$ to form standard generators for a pair of pants. These conditions are in terms of the hyperbolic distance between the axes of $\gamma$ and $\beta$ and the "collar widths" of $\gamma$ and $\beta$. Other writers who have investigated collars include Beardon [Be], Buser [Bu], Halpern [H], Keen [K], Maskit [M], Matelski [Ma], and Randol [R].

A torsion free Fuchsian group $G$ is said to be a pair of pants if $\mathbf{H}^{2} / G$ is topologically a sphere with three holes. The pair of pants is tight if one of the holes is really a puncture (that is, an open neighborhood of it is hyperbolically a punctured disc).

We say that two hyperbolic elements $\gamma$ and $\beta$ form standard generators for a pair of pants $G$ if

i) $\gamma$ and $\beta$ are each primitive boundary elements in $G$ that represent two of the boundary geodesics on $\mathbf{H}^{2} / G$ with $\langle\gamma, \beta\rangle=G$, and

ii) after possibly changing the orientations of $\gamma$ and $\beta$ so that their axes lie to the right of each other, the product $\beta \gamma$ is a primitive boundary element of $G$ which represents the third boundary component (or equivalently, since $\beta \gamma$ is conjugate to $\gamma \beta$ in $G, \beta \gamma$ is a primitive boundary element which represents the third boundary component).

If we think of $\gamma$ and $\beta$ as being elements of the fundamental group of the surface, then the definition essentially says that $\gamma$ and $\beta$ are generators for the fundamental group each of which is freely homotopic to a boundary geodesic with the product $\beta \gamma$ being freely homotopic to the third boundary geodesic.

We note that this definition is symmetric in the sense that if $\gamma$ and $\beta$ are standard generators then so are $\beta$ and $\gamma$. We write $(\gamma, \beta)$ for a pair of standard generators.

Define the collar width of a hyperbolic element to be the quantity,

$$
c(\gamma)=\log \operatorname{coth} T(\gamma) / 4
$$

where $T(\gamma)$ is the translation length of $\gamma$.

Main theorem. Suppose $\gamma$ and $\beta$ are hyperbolic elements. Let $d$ be the hyperbolic distance between the axes of $\gamma$ and $\beta$. Then $(\gamma, \beta)$ form standard generators for a pair of pants if and only if

$$
c(\gamma)+c(\beta) \leq d
$$


with equality if and only if the pair of pants is tight.

Furthermore, $d$ projects to the distance between the boundary geodesics which correspond to $\gamma$ and $\beta$, and the numbers $c(\gamma)$ and $c(\beta)$ determine the widths of disjoint collars on the quotient surface $\mathbf{H}^{2} /\langle\gamma, \beta\rangle$ (see Figure 1).

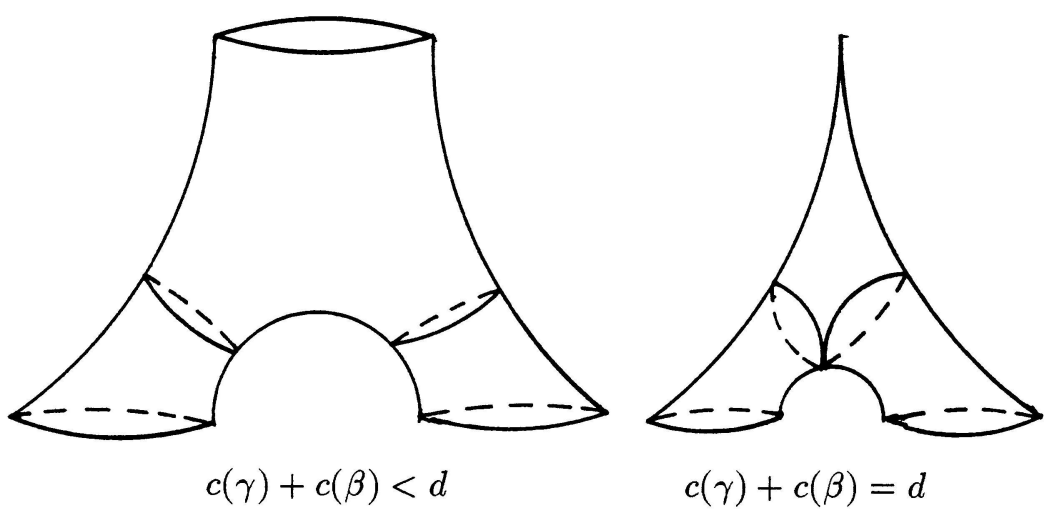

Figure 1.

We remark that for any pair of pants there always exist generators that are not standard. For example consider a standard set of generators $(\gamma, \beta)$ for a pair of pants $G$. Now form a new set of generators for $G$ given by $\left\langle\gamma, \gamma^{2} \beta\right\rangle$. These generators are not standard since $\gamma^{2} \beta$ is not simple.

We introduce some terminology in Section 0. In Section 1 we show that inequality $(*)$ is necessary and sufficient for a standard set of generators. In Section 2 we show that the distance $d$ projects to the quotient, and finally in Section 3 we finish the proof of the main theorem. Some corollaries to the main theorem are listed in Section 4.

The inequality $(*)$ appears with a different form in the work of Seppälä and Sorvali [S-S]. The main theorem appears in the author's Ph.D. thesis [Ba].

I would like to thank my mentor Bernard Maskit for his constant encouragement and invaluable advice.

0. Recall that a Fuchsian group is a discrete subgroup of the orientation preserving isometries of the hyperbolic plane $\mathbf{H}^{2}$. We use $\varrho(\cdot, \cdot)$ to denote the hyperbolic metric. An element $\gamma$ of the orientation preserving isometries is said to be hyperbolic if it fixes two points on the boundary of the hyperbolic plane. In this case $\gamma$ leaves invariant a unique geodesic $A(\gamma)$ called the axis of $\gamma$. An element which only fixes one point on the boundary of the hyperbolic plane is said to be parabolic. A boundary element of a Fuchsian group is either a parabolic element or a hyperbolic element whose axis bounds an interval of discontinuity. 
The hyperbolic distance between two disjoint geodesics in $\overline{\mathbf{H}}^{2}\left(\mathbf{H}^{2}\right.$ with the circle at infinity) is the distance along the unique common orthogonal segment that joins the two geodesics. The distance is zero if the geodesics intersect.

We say that a set of geodesics bound a free polygon if they bound a common domain and they do not intersect in $\mathbf{H}^{2}$.

1. Suppose $\gamma$ and $\beta$ are hyperbolics with disjoint axes. Let $\sigma_{2}$ be reflection in the common orthogonal of these axes. Next construct the reflections $\sigma_{3}=\beta \sigma_{2}$ and $\sigma_{1}=\sigma_{2} \gamma$ (see Figure 2). The distance between the $\sigma_{i}$ and $\sigma_{j}$ reflection circles is denoted by $\varrho\left(\sigma_{i}, \sigma_{j}\right)$.

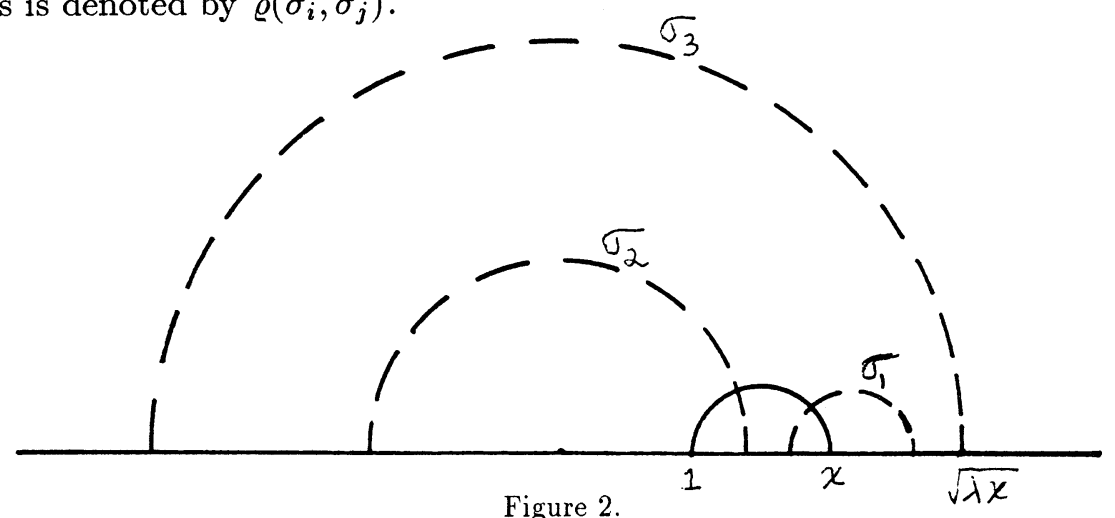

Lemma 1. Let $\gamma$ and $\beta$ be hyperbolic elements with axes $[1, x]$ and $[0, \infty]$ respectively having repelling fixed points $x$ and 0 . Let $\sigma_{i}$ be as above. Define $\sigma_{1}^{\prime}$ to be reflection in the geodesic orthogonal to the axis of $\gamma$ and having the same right endpoint as $\sigma_{3}$. Then the $\sigma_{i}$ reflection circles bound a free polygon if and only if the following inequalities are satisfied

$$
d>c(\beta)
$$

and

$$
T(\gamma) \geq 2 \varrho\left(\sigma_{2}, \sigma_{1}^{\prime}\right)
$$

With equality in (II) if and only if $\sigma_{1}=\sigma_{1}^{\prime}$.

Proof of Lemma 1. First suppose the $\sigma_{i}$ reflection circles bound a free polygon. Since $\beta=\sigma_{3} \sigma_{2}$, we have $\beta A(\gamma) \cap A(\gamma)=\emptyset$. Now $\beta$ has the form $\beta(z)=\lambda z$ for $\lambda>1$; thus $\lambda>x$.

Observe that $x$ is uniquely determined by $d$, and in fact a simple computation yields the formula

$$
x=\left(\frac{e^{d}+1}{e^{d}-1}\right)^{2} .
$$


Hence $d$ is also uniquely determined by $x$.

Writing $\lambda$ as $e^{T(\beta)}$, the inequality $\lambda>x$ translated into hyperbolic terms becomes

$$
e^{T(\beta)}>\left(\frac{e^{d}+1}{e^{d}-1}\right)^{2}
$$

and after some easy manipulations we see that this is precisely inequality (I).

Since the translation length of $\gamma$ is twice the distance between the $\sigma_{1}$ and $\sigma_{2}$ reflection circles, and since $\sigma_{1}^{\prime}$ separates $\sigma_{1}$ from $\sigma_{2}$, we have

$$
T(\gamma) \geq 2 \varrho\left(\sigma_{2}, \sigma_{1}^{\prime}\right)
$$

Furthermore, the fact that two geodesics perpendicular to the same geodesic are either equal or disjoint implies that $T(\gamma)=2 \varrho\left(\sigma_{2}, \sigma_{1}^{\prime}\right)$ if and only if $\sigma_{1}=\sigma_{1}^{\prime}$. This verifies the necessity of (II).

For the converse, assume inequalities (I) and (II) hold. Recall that inequality (I) (by the above arguments) is equivalent to $\lambda>x$ and hence, since by a simple calculation the $\sigma_{3}$ reflection circle has radius $\lambda$, the $\sigma_{3}$ reflection circle does not intersect the axis of $\gamma$ (see Figure 2).

Inequality (II) guarantees that

$$
\varrho\left(\sigma_{2}, \sigma_{1}\right) \geq \varrho\left(\sigma_{2}, \sigma_{1}^{\prime}\right) .
$$

Thus the $\sigma_{1}$ reflection circle is interior to the $\sigma_{1}^{\prime}$ reflection circle. We conclude that the $\sigma_{i}$ reflection circles bound a free polygon. This finishes the proof of Lemma 1. ㅁ

We can restate inqualities (I) and (II) in terms of the collar widths of $\gamma$ and $\beta$.

Lemma 2. Inequalities (I) and (II) are equivalent to

$$
c(\gamma)+c(\beta) \leq d,
$$

with equality if and only if equality in (II).

Proof of Lemma 2. We start with $c(\beta)+c(\gamma) \leq d$. To verify (I) simply observe that $c(\gamma)>0$ and $c(\gamma)+c(\beta) \leq d$ imply that $d>c(\beta)$. Next rewrite $c(\beta)+c(\gamma) \leq d$ as

$$
\log \frac{e^{\frac{1}{2} T(\beta)}+1}{e^{\frac{1}{2} T(\beta)}-1}+\log \frac{e^{\frac{1}{2} T(\gamma)}+1}{e^{\frac{1}{2} T(\gamma)}-1} \leq d .
$$

Exponentiating on both sides and clearing denominators we have

$$
\left(e^{\frac{1}{2} T(\beta)}+1\right)\left(e^{\frac{1}{2} T(\gamma)}+1\right) \leq e^{d}\left(e^{\frac{1}{2} T(\beta)}-1\right)\left(e^{\frac{1}{2} T(\gamma)}-1\right) .
$$


After multiplying and gathering terms we obtain

$$
\left[-e^{d}\left(e^{\frac{1}{2} T(\beta)}-1\right)+\left(e^{\frac{1}{2} T(\beta)}+1\right)\right] e^{\frac{1}{2} T(\gamma)}+\left(e^{\frac{1}{2} T(\beta)}+1\right) \leq-e^{d}\left(e^{\frac{1}{2} T(\beta)}-1\right) .
$$

Note that $\left[-e^{d}\left(e^{\frac{1}{2} T(\beta)}-1\right)+\left(e^{\frac{1}{2} T(\beta)}+1\right)\right]<0$, since $d>c(\beta)$.

Solving for $T(\gamma)$ we find that

$$
T(\gamma) \geq 2 \log \frac{-e^{d}\left(e^{\frac{1}{2} T(\beta)}-1\right)-\left(e^{\frac{1}{2} T(\beta)}+1\right)}{-e^{d}\left(e^{\frac{1}{2} T(\beta)}-1\right)+\left(e^{\frac{1}{2} T(\beta)}+1\right)}
$$

and hence, by some straightforward rearranging we have

$$
T(\gamma) \geq 2 \log \frac{e^{\frac{1}{2} T(\beta)}\left(e^{d}+1\right)-\left(e^{d}-1\right)}{e^{\frac{1}{2} T(\beta)}\left(e^{d}-1\right)-\left(e^{d}+1\right)} .
$$

Now it is a simple, albeit tedious, computation to make the substitutions $T(\beta)=$ $\log \lambda$ and $d=\log ((\sqrt{x}+1) /(\sqrt{x}-1))$ to show that

$$
T(\gamma) \geq 2 \log \left(\frac{\sqrt{\lambda x}-1}{\sqrt{\lambda}-\sqrt{x}}\right)
$$

Finally we would like to show that the right side of the above inequality is precisely $2 \varrho\left(\sigma_{2}, \sigma_{1}^{\prime}\right)$. To compute this distance we normalize the axis of $\gamma$ by an element $A$ of $\operatorname{PSL}(2, \mathbf{R})$ which takes 1 to $0, \sqrt{x}$ to 1 , and $x$ to $\infty$. Note that a simple computation shows that the right endpoint of $\sigma_{1}^{\prime}$ (which is the same as the right endpoint of $\left.\sigma_{3}\right)$ is $\sqrt{\lambda x}$ (see Figure 3 ). Thus,

$$
\varrho\left(\sigma_{2}, \sigma_{1}^{\prime}\right)=\log |A(\sqrt{\lambda x})|=\log \left(\frac{\sqrt{\lambda x}-1}{\sqrt{\lambda}-\sqrt{x}}\right)
$$

This verifies inequality (II). Obviously equality is preserved at each step and all of these computations are reversible.

Proof that $(*)$ is necessary and sufficient. Note that if the axes of $\gamma$ and $\beta$ intersect in $\overline{\mathbf{H}}^{2}$, then $d=0$ and the theorem is trivially correct. Thus, for the rest of the proof we consider $\gamma$ and $\beta$ to have disjoint axes.

Assume $(\gamma, \beta)$ are standard generators for a pair of pants. If necessary change the orientations of $\gamma$ and $\beta$ so that their axes are oriented to the right of each other. Normalize the group $\langle\gamma, \beta\rangle$, by conjugating in $\operatorname{PSL}(2, \mathbf{R})$, so that the axis 


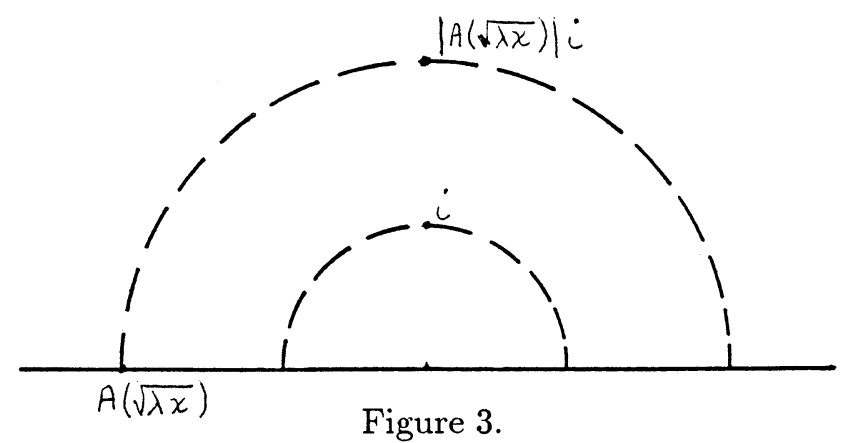

of $\beta$ is $[0, \infty]$, the axis of $\gamma$ is $[1, x]$, and the axis of $\beta \gamma$ lies to the right of the axis of $\gamma$.

The group $\langle\gamma, \beta\rangle$ is a subgroup of index two in the reflection group generated by reflection in the common orthogonals joining the three axes. Denote these reflections by $\sigma_{1}, \sigma_{2}$, and $\sigma_{3}$ as in Figure 2; thus $\gamma=\sigma_{2} \sigma_{1}, \beta=\sigma_{3} \sigma_{2}$, and $(\beta \gamma)^{-1}=\sigma_{1} \sigma_{3}$.

Observe that since the group $\langle\gamma, \beta\rangle$ contains no elliptics, the $\sigma_{i}$ reflection circles must be disjoint and hence bound a free polygon. Thus we can apply Lemmas 1 and 2 to conclude the necessity of inequality $(*)$ in the main theorem.

To prove the sufficiency, suppose $\gamma$ and $\beta$ are hyperbolic elements so that $c(\beta)+c(\gamma) \leq d$ or equivalently (I) and (II) are satisfied. Normalize in PSL $(2, \mathbf{R})$ and change orientations if necessary so that $\beta$ has repelling fixed point 0 , attracting fixed point $\infty$, and $\gamma$ has attracting fixed point 1 (let $x$ be the repelling fixed point of $\gamma)$. Hence $\beta(z)=\lambda z$, for $\lambda>1$.

Construct the common orthogonal to the geodesics $[0, \infty]$ and $[1, x]$ and call reflection in this geodesic $\sigma_{2}$. Set $\sigma_{3}=\beta \sigma_{2}$ and $\sigma_{1}=\sigma_{2} \gamma$. Then by Lemma 1 the $\sigma_{i}$ reflection circles bound a free polygon (see Figure 2).

The group $\left\langle\sigma_{1}, \sigma_{2}, \sigma_{3}\right\rangle$ acts discontinuously on $\mathbf{H}^{2}$. To see this, observe that the free polygon bounded by the $\sigma_{i}$ reflection circles and the boundary of $\mathbf{H}^{2}$ is a fundamental polygon for $\left\langle\sigma_{1}, \sigma_{2}, \sigma_{3}\right\rangle$.

Now consider the orientation preserving subgroup $\langle\gamma, \beta\rangle$ of $\left\langle\sigma_{1}, \sigma_{2}, \sigma_{3}\right\rangle .\langle\gamma, \beta\rangle$ is an index two subgroup of a discontinuous group, hence $\langle\gamma, \beta\rangle$ acts discontinuously on $\mathbf{H}^{2}$. Furthermore it is not hard to see that $\langle\gamma, \beta\rangle$ is a pair of pants with standard generators $(\gamma, \beta)$, where $\eta=\gamma^{-1} \beta^{-1}$ is either a boundary hyperbolic or a parabolic element.

Finally, by Lemmas 1 and 2 , note that equality holds for $(*)$ if and only if the $\sigma_{i}$ reflection circles bound a free polygon with $\sigma_{i}=\sigma_{i}^{\prime}$; that is $(\gamma, \beta)$ are standard generators for a pair of tight pants. $\square$

This proves the first half of the main theorem.

2. Suppose $(\gamma, \beta)$ are standard generators for a pair of pants. Without loss of generality we can think of $\gamma$ and $\beta$ as being normalized so that their axes are 
as in Figure 2 and the $\sigma_{i}$ are, as before, reflections in common orthogonals where $\beta=\sigma_{3} \sigma_{2}$ and $\gamma=\sigma_{2} \sigma_{1}$. We define the interior of the $\sigma_{i}$ reflection circle to be the connected component of $\mathbf{H}^{2}-\left\{\sigma_{i}\right.$ reflection circle $\}$ which does not contain the other reflection circles.

The projections of the axes of $\gamma$ and $\beta$ are boundary geodesics and since the quotient surface is complete, the hyperbolic distance between these closed geodesics is realized by a geodesic line segment orthogonal to the boundary geodesics. We would like to show that this geodesic segment is the image of the common orthogonal segment between the axes of $\gamma$ and $\beta$. Hence this would show that the distance between the axes of standard generators of a pair of pants projects to the distance between the boundary geodesics that represent the standard generators. It is in fact enough to prove the following proposition.

Proposition. We have for all $\tau \in\left\langle\sigma_{1}, \sigma_{2}, \sigma_{3}\right\rangle$

$$
\varrho(\tau A(\gamma), A(\beta)) \geq \varrho(A(\gamma), A(\beta)) .
$$

To prove this, first observe that $\sigma_{1}$ and $\sigma_{2}$ keep the axis of $\gamma$ invariant. Hence, we can assume that $\tau$ has the form $\sigma_{i_{n}} \cdots \sigma_{3}$ and no two successive factors are the same. At this point, the proposition follows by inducting on the following lemma.

Lemma. Let $C$ be a geodesic which is contained in the interior of the $\sigma_{i}$ reflection circle and whose endpoints lie on the positive real axis. Furthermore suppose that the euclidean line passing through the origin and tangent to $C$ has slope $m$. Then $C$ gets sent by $\sigma_{j}(j \neq i)$ to a geodesic in the interior of the $\sigma_{j}$ reflection circle having a tangent line through the origin with slope no bigger than $m$. The slope is smaller if and only if $\sigma_{j}=\sigma_{1}$.

In essence, the lemma says that as we apply more and more reflections to the axis of $\gamma$, its distance from the axis of $\beta$ can only get bigger.

Proof of Lemma. Clearly reflection by $\sigma_{j}$ sends $C$ to the interior of the $\sigma_{j}$ reflection circle. Also the tangent line to $C$ is invariant under $\sigma_{2}$ and $\sigma_{3}$. In particular the slope of the tangent line remains the same. Hence the only case we need to consider is when $\sigma_{j}=\sigma_{1}$ and $C$ lies either in the $\sigma_{2}$ or $\sigma_{3}$ reflection circle. Assume for the sake of argument that $C$ lies in the $\sigma_{3}$ reflection circle (the argument for $\sigma_{2}$ is similar).

Draw the line $L$ tangent to $C$ and passing through the center of the $\sigma_{1}$ reflection circle. Notice that $L$ lies below the tangent line to $C$ through the origin and $\sigma_{1}$ keeps $L$ invariant. Since $\sigma_{1} C$ lies below the tangent line $L$, the slope of the line $L$ tangent to $\sigma_{1} C$ and passing through the origin is smaller than the slope of the tangent line of $C$ through the origin. $\square$

3. Let $\beta$ be a hyperbolic element with axis $A(\beta)$. The canonical region for $\beta$ is

$$
\mathbf{R}(\beta)=\left\{z \in \mathbf{H}^{2}: \varrho(z, A(\beta))<c(\beta)\right\} .
$$


See Beardon [B] for an equivalent geometric definition of $\mathbf{R}(\beta)$.

We say that a subset $X$ of the hyperbolic plane is precisely invariant under the subgroup $\Gamma$ of $G$ if

$$
\gamma X=X \quad \text { for all } \quad \gamma \in \Gamma
$$

and

$$
g X \cap X=\emptyset \quad \text { for all } \quad g \in G-\Gamma .
$$

If $X$ is open this says exactly that $X / \Gamma$ isometrically imbeds into $\mathbf{H}^{2} / G$.

In this section, we would like to show that the canonical region of a primitive simple hyperbolic projects to a collar on the surface. In order to accomplish this we will need the following lemma.

Lemma. Suppose $\gamma$ and $\beta$ are simple hyperbolic elements with disjoint axes in a torsion free Fuchsian group. Then $(\gamma, \beta)$ are standard generators for a pair of pants.

Proof. Normalize $\gamma$ and $\beta$ as we previously did in Figure 1 and also draw the $\sigma_{i}$ reflection circles as before. Since $\sigma$ is simple the $\sigma_{3}$ reflection does not intersect the axis of $\gamma$. Similarly, since $\beta$ is simple the $\sigma_{1}$ reflection circle does not intersect the axis of $\beta$. Moreover the $\sigma_{3}$ and $\sigma_{1}$ reflection circles are disjoint for otherwise their product would be elliptic. Hence the $\sigma_{i}$ reflection circles bound a free polygon, and thus by Lemmas 1 and $2, \gamma$ and $\beta$ satisfy the inequality

$$
c(\gamma)+c(\beta) \leq d .
$$

We conclude from the main theorem that $\gamma$ and $\beta$ are standard generators for a pair of pants. $\square$

Proposition. Let $\beta$ be a primitive simple hyperbolic in a torsion free Fuchsian group $G$. Then $\mathbf{R}(\beta)$ is precisely invariant under $\langle\beta\rangle$ in $G$.

Proof. Clearly all powers of $\beta$ keep $\mathbf{R}(\beta)$ invariant. Next suppose there exists some $g \in G-\langle\beta\rangle$ so that $g \mathbf{R}(\beta) \cap \mathbf{R}(\beta) \neq \emptyset$, that is $\mathbf{R}\left(g \beta g^{-1}\right) \cap \mathbf{R}(\beta) \neq \emptyset$. This implies that

$$
c\left(g \beta g^{-1}\right)+c(\beta)>\varrho\left(A\left(g \beta g^{-1}\right), A(\beta)\right) .
$$

On the other hand, $\beta$ being simple implies that the axes of $g \beta g^{-1}$ are $\beta$ are disjoint. Furthermore, $g \beta g^{-1}$ is simple in $G$ since it is conjugate to $\beta$. Thus by the above lemma $\left(g \beta g^{-1}, \beta\right)$ are standard generators for a pair of pants and hence

$$
c\left(g \beta g^{-1}\right)+c(\beta) \leq \varrho\left(A\left(g \beta g^{-1}\right), A(\beta)\right) .
$$

We have reached a contradiction. $\square$

Now we can conclude that the canonical regions for $\beta$ and $\gamma$ project to disjoint collars on the pair of pants $\langle\gamma, \beta\rangle$. For otherwise if the collars did intersect, the distance $d$ from the projections $\pi A(\gamma)$ to $\pi A(\beta)$ ( $\pi$ is the projection map to the quotient) would have to be less than that of $c(\gamma)+c(\beta)$. Clearly a contradiction. This completes the proof of the main theorem. 
4. In this section we list two corollaries to the main theorem. The first is a collar lemma.

Corollary. Two disjoint simple closed geodesics $\gamma$ and $\beta$ on a hyperbolic Riemann surface have disjoint collars of width $c(\gamma)$ and $c(\beta)$ respectively. Furthermore, this collar lemma is sharp in the sense that if the collar width of a simple closed geodesic were any bigger, then there exists a surface where the collars intersect.

Proof. To see that the lemma is sharp, simply observe that on a tight pair of pants the boundaries of the collars meet tangentially. Hence if they were of any bigger width they would intersect.

Next to prove that the collars are disjoint, suppose not. Then, using the canonical isomorphism, there exist lifts of $\gamma$ and $\beta$ to simple hyperbolic elements with disjoint axes in a torsion free Fuchsian group so that the canonical regions of these hyperbolic elements intersect. Call the lifts $\gamma$ and $\beta$.

On the other hand, we know that $(\gamma, \beta)$ form standard generators for a pair of pants, and hence (by the main theorem) their canonical regions are disjoint. We have arrived at a contradiction.

We remark that this is a new proof of a collar lemma due to Buser [Bu]. Different versions of this lemma have appeared in $[\mathrm{H}],[\mathrm{K}],[\mathrm{M}],[\mathrm{Ma}]$, and $[\mathrm{R}]$.

Let $\alpha$ be a curve on a hyperbolic Riemann surface that starts and ends in a simple closed geodesic $\beta$ (that is, $\alpha:[0,1] \rightarrow S$ is continuous and $\alpha(0) \in \beta$ and $\alpha(1) \in \beta$ ). We say that $\alpha$ has nontrivial relative free homotopy if it is freely homotopic to a common orthogonal segment joining $\beta$ to itself, where the homotopy leaves the endpoints of $\alpha$ in $\beta$. We denote the hyperbolic length of a curve $\alpha$ by $L(\alpha)$. We have the following corollary.

Corollary. Let $\beta$ be a simple closed geodesic in a hyperbolic surface $S$, and let $\alpha$ be a curve that starts and ends in $\beta$ and has nontrivial relative free homotopy. Then $2 c(\beta) \leq L(\alpha)$.

Proof. Let $G$ be a Fuchsian group representing $S . \beta$ and $\alpha$ determine two conjugate simple hyperbolic elements in $G$ with disjoint axes that are joined by the lift of $\alpha$. Call these hyperbolic elements $\beta$ and $\beta^{\prime}$. Let $d$ be the hyperbolic distance from the axis of $\beta$ to the axis of $\beta^{\prime}$. Then since $\left(\beta, \beta^{\prime}\right)$ form standard generators for a pair of pants we have

$$
2 c(\beta)=c(\beta)+c\left(\beta^{\prime}\right) \leq d \leq L(\alpha) \text {. }
$$

Notice that if $\alpha$ is a closed geodesic that intersects $\beta$ transversely we arrive at the well known result that the length $L(\alpha) \rightarrow \infty$ as $L(\beta) \rightarrow 0$. 


\section{References}

[Ba] Basmajian, A.: Hyperbolic invariants for infinitely generated Fuchsian groups. - Ph.D. thesis, Stony Brook, 1987.

[Be] Beardon, A.: The geometry of discrete groups. - Springer-Verlag, New York, 1983.

[Bu] Buser, P.: The collar theorem and examples. - Manuscripta Math. 25, 1978, 349-357.

[H] Halpern, N.: A proof of the collar lemma. - Bull. London Math. Soc. 13, 1981, 141-144.

[K] KeEn, L.: Collars on Riemann surfaces, discontinuous groups and Riemann surfaces. Ann. of Math. Studies 79. Princeton University Press, Princeton, NJ, 1974, 263-268.

[M] Maskit, B.: Comparison of hyperbolic and extremal lengths. - Ann. Acad. Sci. Fenn. Ser. A I Math. 10, 1985, 381-386.

[Ma] Matelski, J.P.: A compactness theorem for Fuchsian groups of the second kind. - Duke Math. J. 43, 1976, 829-840.

[R] Randol, B.: Cylinders in Riemann surfaces. - Comment. Math. Helv. 54, 1979, 1-5.

[S-S] SEPPÄLÄ, M., and T. SoRvali: Parametrization of Teichmüller spaces by geodesic length functions. - In Holomorphic functions and moduli, Volume II; edited by D. Drasin. Springer-Verlag, New York, 1988.

University of Oklahoma

Department of Mathematics

Norman, OK 73019

U.S.A.

Received 2 March 1989 